\title{
Postoperative survival and functional outcomes for patients with metastatic gynecological cancer to the spine: case series and review of the literature
}

\author{
*Ann Liu, BS, Eric W. Sankey, BS, C. Rory Goodwin, MD, PhD, Thomas A. Kosztowski, MD, \\ Benjamin D. Elder, MD, PhD, Ali Bydon, MD, Timothy F. Witham, MD, Jean-Paul Wolinsky, MD, \\ Ziya L. Gokaslan, MD, and Daniel M. Sciubba, MD \\ Department of Neurosurgery, Johns Hopkins Hospital, Baltimore, Maryland
}

\begin{abstract}
OBJECTIVE Spinal metastases from gynecological cancers are rare, with few cases reported in the literature. In this study, the authors examine a series of patients with spinal metastases from gynecological cancer and review the literature.
\end{abstract}

METHODS The cases of 6 consecutive patients who underwent spine surgery for metastatic gynecological cancer between 2007 and 2012 at a single institution were retrospectively reviewed. The recorded demographic, operative, and postoperative factors were reviewed, and the functional outcomes were determined by change in Karnofsky Performance Scale and the American Spine Injury Association (ASIA) score during follow-up. A systematic review of the literature was also performed to evaluate outcomes for patients with similar gynecological metastases to the spine.

RESULTS In this series, details regarding metastatic gynecological cancers to the spine are as follows: 2 patients with cervical cancer (both presented at age 46 years, mean postoperative survival of 32 months), 2 patients with endometrial cancer (mean age of 40 years, mean postoperative survival of 26 months), and 2 patients with leiomyosarcoma (mean age of 44 years, mean postoperative survival of 20 months). All patients presented with pain, and no complications were noted following surgery. All patients with known follow-up had stable or improved neurological outcomes, performance status, and improved pain, without local recurrence of tumor. Overall median survival after diagnosis of metastatic spine lesions for all cases in the literature as well as those treated by the authors was 15 months. When categorized by type, median survival of patients with cervical cancer $(n=2)$, endometrial cancer $(n=26)$, and leiomyosarcoma $(n=16)$ was 32,10 , and 22.5 months, respectively.

CONCLUSIONS Gynecological cancers metastasizing to the spine are rare. In this series, overall survival following diagnosis of spinal metastasis and surgery was 27 months, with cervical cancer, endometrial cancer, and leiomyosarcoma survival being 32,26 , and 20 months, respectively. Combined with literature cases, survival differs depending on primary histology, with decreasing survival from cervical cancer (32 months) to leiomyosarcoma (22.5 months) to endometrial cancer (10 months). Integrating such information with other patient factors may more accurately guide decision making regarding management of such spinal lesions.

http://thejns.org/doi/abs/10.3171/2015.3.SPINE15145

KEY WORDS spine; metastasis; endometrial carcinoma; cervical cancer; leiomyosarcoma; surgery; tumor; gynecological; oncology

ABBREVIATIONS ASIA = American Spine Injury Association; DVT = deep vein thrombosis; KPS = Karnofsky Performance Scale; UTI = urinary tract infection. SUBMITTED February 2, 2015. ACCEPTED March 26, 2015.

INCLUDE WHEN CITING Published online September 11, 2015; DOI: 10.3171/2015.3.SPINE15145.

${ }^{*}$ Ms. Liu and Mr. Sankey contributed equally to this work. 
$\mathrm{I}$ $\mathrm{N}$ the United States, the estimated incidence of gynecological cancer is approximately $11 \%, 32$ with 71,500 new diagnoses and 26,500 deaths each year. ${ }^{7}$ The 3 most common types are uterine $(53 \%)$, ovarian $(25 \%)$, and cervical (14\%). ${ }^{32}$ Management depends on the site and extent of disease but typically involves a combination of surgery, chemoradiation, and hormone therapy. Ovarian cancer carries the poorest prognosis with a 5-year survival of $44.6 \%$ as compared with $67.9 \%$ for cervical cancer and $81.5 \%$ for endometrial cancer. ${ }^{25-27}$ Leiomyosarcoma is a rare, malignant connective tissue tumor originating from smooth muscle cells ${ }^{8}$ and most frequently arises in the uterus, gastrointestinal tract, or retroperitoneum. ${ }^{31}$ Due to its high rate of metastatic recurrence and resistance to radiation and chemotherapy, prognosis is poor.

Metastasis of gynecological cancers varies depending on the type. Cervical cancer, endometrial cancer, and leiomyosarcoma most commonly metastasize to the lung and liver, ${ }^{16,22}$ while ovarian cancer spreads locally within the peritoneum and pelvis. ${ }^{20}$ Bone metastases are seen more commonly in cervical cancer but are infrequent in endometrial cancer and leiomyosarcoma. Among bone metastases, the spine is a common site; however, due to the rarity of this occurrence, surgical management of spinal metastases has not been well described. We retrospectively reviewed the medical records of patients who underwent surgery for spinal metastases of gynecological cancer at our institution and performed a literature review to identify other published reports to obtain more accurate prognostic information on such rare lesions.

\section{Methods \\ Case Series}

After obtaining approval from the institutional review board, a database of patients who underwent spine surgery for metastatic cancer from 2007 to 2012 at our institution was screened, and 6 patients were identified with primary tumors of gynecological origin that metastasized to the spine. Medical, imaging, and operative records for each of these patients were retrospectively reviewed.

Demographic factors, including age, race, smoking history, and comorbidities were reviewed. Additionally, prior cancer history, preoperative interventions, operative approach and techniques, postoperative factors, interventions, adjuvant therapies, functional outcome, and survival were assessed. The prior cancer history included primary tumor histological diagnosis, time from primary diagnosis, history of adjuvant therapies (chemotherapy, radiotherapy, etc.), time to diagnosis of spinal metastasis, and presenting symptoms. Operative factors included indication for surgery, type of surgical procedure, approach, instrumentation, levels involved, vertebrectomy, intraoperative complications, and estimated blood loss. Postoperative factors included need for blood transfusion, hospital length of stay, discharge location, adjuvant treatment, local recurrence, and survival. Functional outcome was determined by change in Karnofsky Performance Scale (KPS) score, and neurological outcome was evaluated by change in the American Spine Injury Association (ASIA) score.

\section{Literature Review}

A review of the literature was performed using PubMed as well as a review of the bibliographies of eligible articles. The search string employed for cervical cancer was ("uterine cervical neoplasms"[MeSH Terms] OR ("uterine"[All Fields] AND "cervical"[All Fields] AND "neoplasms"[All Fields]) OR "uterine cervical neoplasms"[All Fields] OR ("cervix"[All Fields] AND "cancer"[All Fields]) OR “cervix cancer"[All Fields]) AND ("spine"[MeSH Terms] OR "spine"[All Fields]) AND ("neoplasm metastasis"[MeSH Terms] OR ("neoplasm"[All Fields] AND "metastasis"[All Fields]) OR "neoplasm metastasis"[All Fields] OR "metastasis"[All Fields]). The search string for endometrial cancer was ("endometrial neoplasms"'[MeSH Terms] OR ("endometrial"'All Fields] AND "neoplasms"[All Fields]) OR "endometrialneoplasms" [All Fields] OR ("endometrial"[All Fields] AND "cancer" [All Fields]) OR “endometrial cancer"[All Fields]) AND ("spine"[MeSH Terms] OR "spine"[All Fields]) AND ("neoplasm metastasis"[MeSH Terms] OR ("neoplasm"[All Fields] AND "metastasis"[All Fields]) OR "neoplasm metastasis"[All Fields] OR "metastasis"[All Fields]). For Leiomyosarcoma, we used ("leiomyosarcoma" [MeSH Terms] OR "leiomyosarcoma"[All Fields]) AND ("spine" [MeSH Terms] OR "spine"[All Fields]) AND ("neoplasm metastasis"[MeSH Terms] OR ("neoplasm"[All Fields] AND "metastasis"[All Fields]) OR "neoplasm metastasis" [All Fields] OR "metastasis"[All Fields]).

Criteria for inclusion were articles written in English or those having an English translation; articles describing patients with confirmed gynecological leiomyosarcoma, endometrial cancer, or cervical cancer and metastases to the spine; and fully published, peer-reviewed studies including randomized controlled trials, nonrandomized trials, cohort studies, case control studies, case series, and case reports. Criteria for exclusion were articles with no extractable data specific to metastatic spine disease, articles looking at primary spine tumors, and studies of cases with unconfirmed primary tumor pathology.

\section{Statistical Analysis}

Survival statistics and Kaplan-Meier curves were calculated using GraphPad Prism 5.0. Cases from the literature as well as our institution were included. Cases with unknown follow-up or survival times were excluded from the analysis.

\section{Results}

\section{Summary of Cases}

Our series (Table 1) consisted of 2 patients with cervical cancer (both presented at age 46 years, mean postoperative survival of 32 months), 2 patients with endometrial cancer (mean age of 40 years, mean postoperative survival of 26 months), and 2 patients with leiomyosarcoma (mean age of 44 years, mean postoperative survival of 20 months). There were no patients with metastatic ovarian cancer to the spine. None of the patients had other medical comorbidities, although 3 patients had a smoking history. Only 1 patient had undergone prior radiation therapy for her primary tumor; none of the women received preop- 


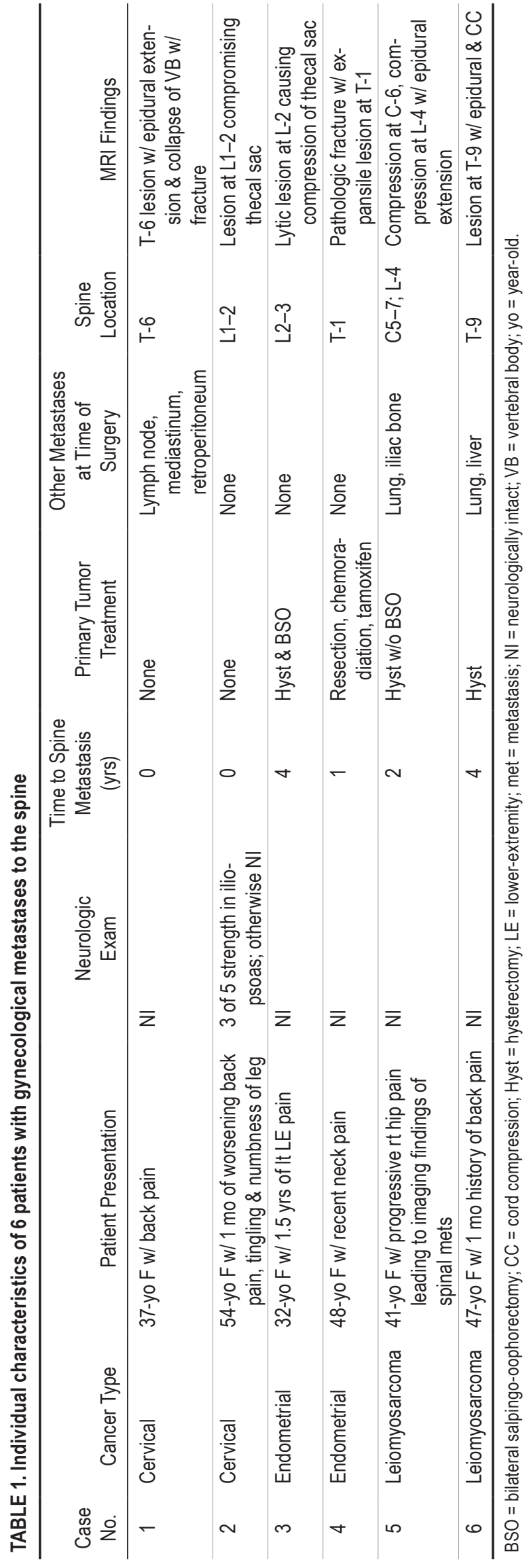

erative chemotherapy or neoadjuvant radiotherapy to the spine. All patients presented with focal spine pain, with 1 patient having concurrent paresthesias and another having concurrent motor weakness and gait difficulties. All patients had a preoperative ASIA score of D or E.

Metastases were most commonly located in the thoracic spine $(\mathrm{n}=5,83 \%)$ and were also seen in the cervical spine $(\mathrm{n}=1,17 \%)$ and lumbar spine $(\mathrm{n}=3,50 \%)$. Indications for surgery included cord compression alone $(\mathrm{n}=2$, $33 \%)$, instability and cord compression $(\mathrm{n}=3,50 \%)$, and severe pain $(\mathrm{n}=1,17 \%)$. The 6 patients underwent a total of 8 surgeries, and all underwent tumor resection and spinal fusion (Table 2). Four patients underwent a single surgery. One leiomyosarcoma patient underwent an additional staged surgery for new spinal metastases, and 1 patient with cervical cancer underwent a staged procedure. Median blood loss for cervical cancer, endometrial cancer, and leiomyosarcoma was 200, 2425, and $550 \mathrm{ml}$, respectively. No intraoperative complications were noted, and postoperative complications included DVT, atelectasis, UTI, and intractable pain. No patients experienced instrument failure, required postoperative blood transfusion, or required revision. Median hospital stay was 7 days (range 3-9 days). Four patients were discharged to home and 2 were discharged to inpatient rehabilitation.

All 5 patients with follow-up had improvement or complete resolution of their pain postoperatively. Baseline KPS and ASIA scores remained stable or improved in 5 patients (83\%) but was unknown in 1 patient at last followup (patient died 6 months after surgery). One patient received no postoperative adjuvant treatment and 5 patients received adjuvant radiation. As seen in Table 3, the total dose ranged from $3000 \mathrm{cGy}$ to $3750 \mathrm{cGy}$. No patients had local recurrence of tumor as evaluated on MRI. Mean postoperative survival for leiomyosarcoma, endometrial cancer, and cervical cancer was 20,26, and 32 months, respectively, with 2 patients (endometrial, cervical) alive at 28 and 37 months postoperatively (Table 4).

\section{Literature Review}

For patients with cervical cancer, a total of 3 articles described a total of 13 cases of metastasis to the spine (Table 5). Median age at presentation was 53 years (range 30-84 years). The lumbar spine was the most common location of metastasis (10 of 13). Treatment and time to follow-up was reported for only 1 patient, who received chemoradiation and survived a few months. Only $6(46 \%)$ of 13 patients were alive at last follow-up.

For patients with endometrial cancer, 6 articles described a total of 25 cases of metastasis to the spine (Table 6). Median age at presentation was 62 years (range $47-80$ years). Of the 16 cases with described metastasis location, the most common location involved was the thoracic spine (7 of 16), followed by the sacrum (6 of 16). Two patients were treated surgically: 1 patient underwent a sacrectomy through a posterior approach from S2, with en bloc excision of metastasis, and the other patient underwent T12 vertebrectomy and anterior spinal fusion. Of the $24 \mathrm{pa}-$ tients with known survival, median survival was 9 months (range 1-199 months). The 1-year and 5-year survival rates were $38 \%$ and $8.3 \%$, respectively. Only 4 patients (16\%) 

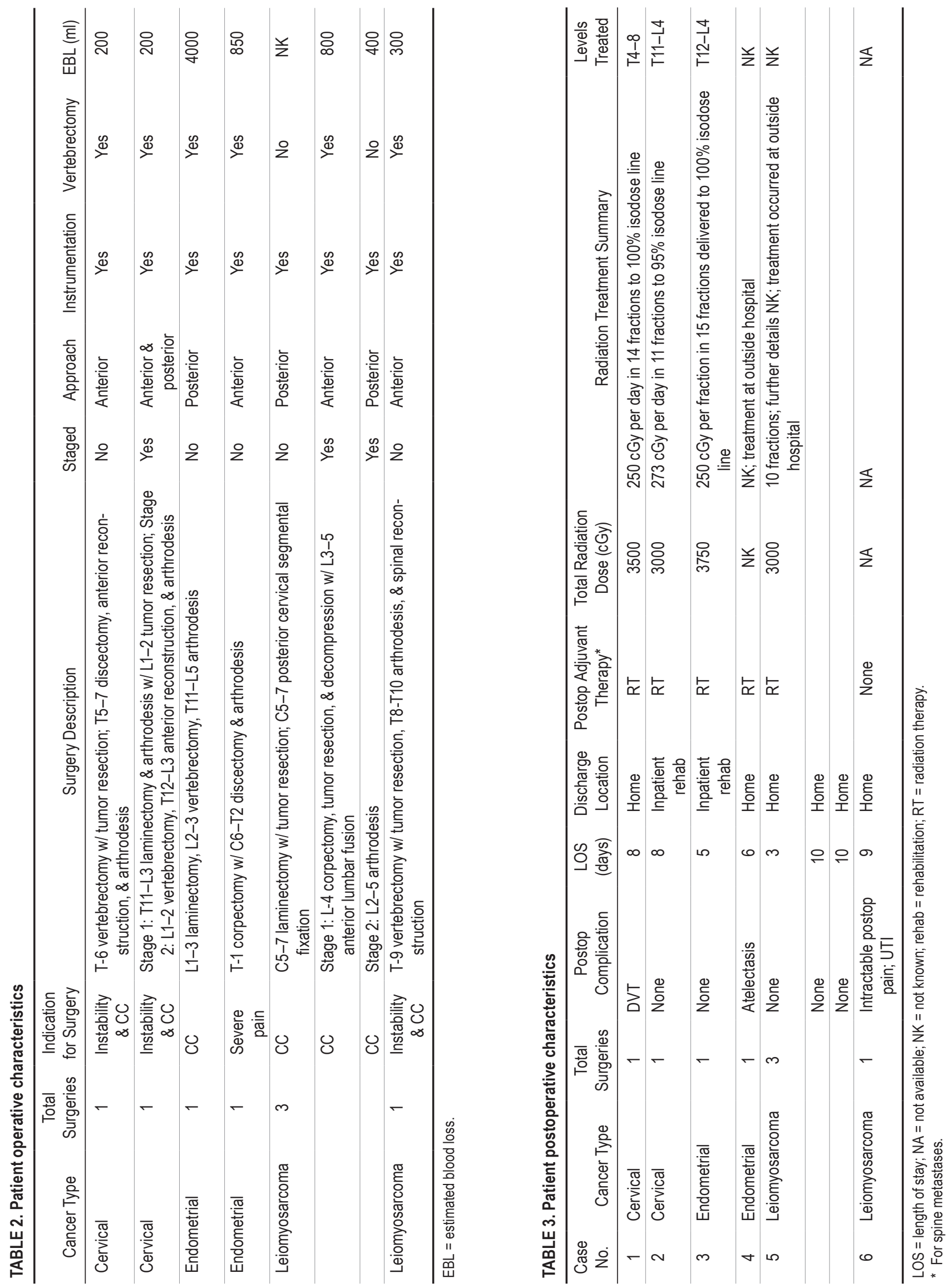


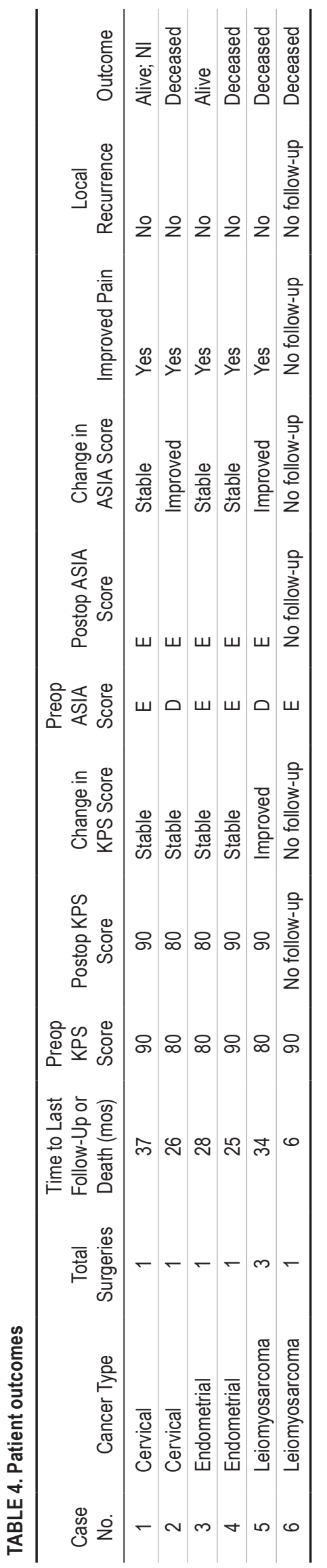

were alive at last follow-up, including the 2 patients who were treated surgically.

For patients with leiomyosarcoma of gynecological origin, a total of 11 articles describing 18 cases of spine metastasis were found (Table 7). Median age at presentation was 49 years (range 35-64 years). The most common location involved was the thoracic spine (10 of 18), followed by the lumbar spine (9 of 18). Thirteen patients were treated surgically. Four patients developed postoperative recurrence in the spine. Of the 14 patients with known survival, the median survival was 22.5 months (range 3.3-120 months). The 1-year and 5-year survival rates were $64 \%$ and $21 \%$, respectively. Ten patients were alive at last follow-up.

\section{Patient Survival}

Among our cases and the cases found in the literature, 2 cases of cervical cancer, 26 cases of endometrial cancer, and 16 cases of leiomyosarcoma had known survival after diagnosis of spinal metastasis. Of note, for cervical cancer, our case series is the first to report known survival times for spinal metastasis; the prior 13 cases found in the literature did not report survival. Overall median survival for all cases was 15 months (Fig. 1). Based on our cases and the cases found in the literature, median survival of cervical cancer, endometrial cancer, and leiomyosarcoma patients was 32, 10, and 22.5 months, respectively (Fig. 2).

\section{Discussion}

In our series, overall survival following spine surgery for such lesions was 27 months, with cervical cancer, endometrial cancer, leiomyosarcoma survival being 32,26 , and 20 months, respectively. Combined with cases from the literature, median survival of cervical cancer $(n=2)$, endometrial cancer $(n=26)$, and leiomyosarcoma $(n=16)$ patients was 32,10 , and 22.5 months, respectively. Although surgery for leiomyosarcoma spine metastases has shown benefit in improving pain and neurological function, ${ }^{9,38}$ similar to other spinal metastases, ${ }^{6,13}$ to the best of our knowledge, the surgical outcomes of patients with cervical or endometrial metastases to the spine has not been reported. Here, we present a case series of patients who underwent resection of a gynecological metastasis spinal lesion and combine our series with all reported cases in the literature.

\section{Surgery for Spinal Metastasis From Cervical Cancer}

For cervical cancer, the reported prevalence of spine metastases ranges from $0.6 \%$ to $6.5 \%$, with the lumbar spine being the most common site. ${ }^{4,10,12,19,23,28,35}$ Once diagnosed with bone metastases from cervical cancer, treatment is focused on palliation as prognosis is poor, with the majority of patients dying within 1 year. ${ }^{23}$ Interestingly, the primary tumor of both of our patients with cervical cancer was diagnosed after presenting with spine metastases. They survived an average of 32 months; however, their survival is difficult to compare with prior studies, which examine length of survival of all patients with bone metastases rather than survival of those with spine metastases alone. In these studies, survival from discovery of 


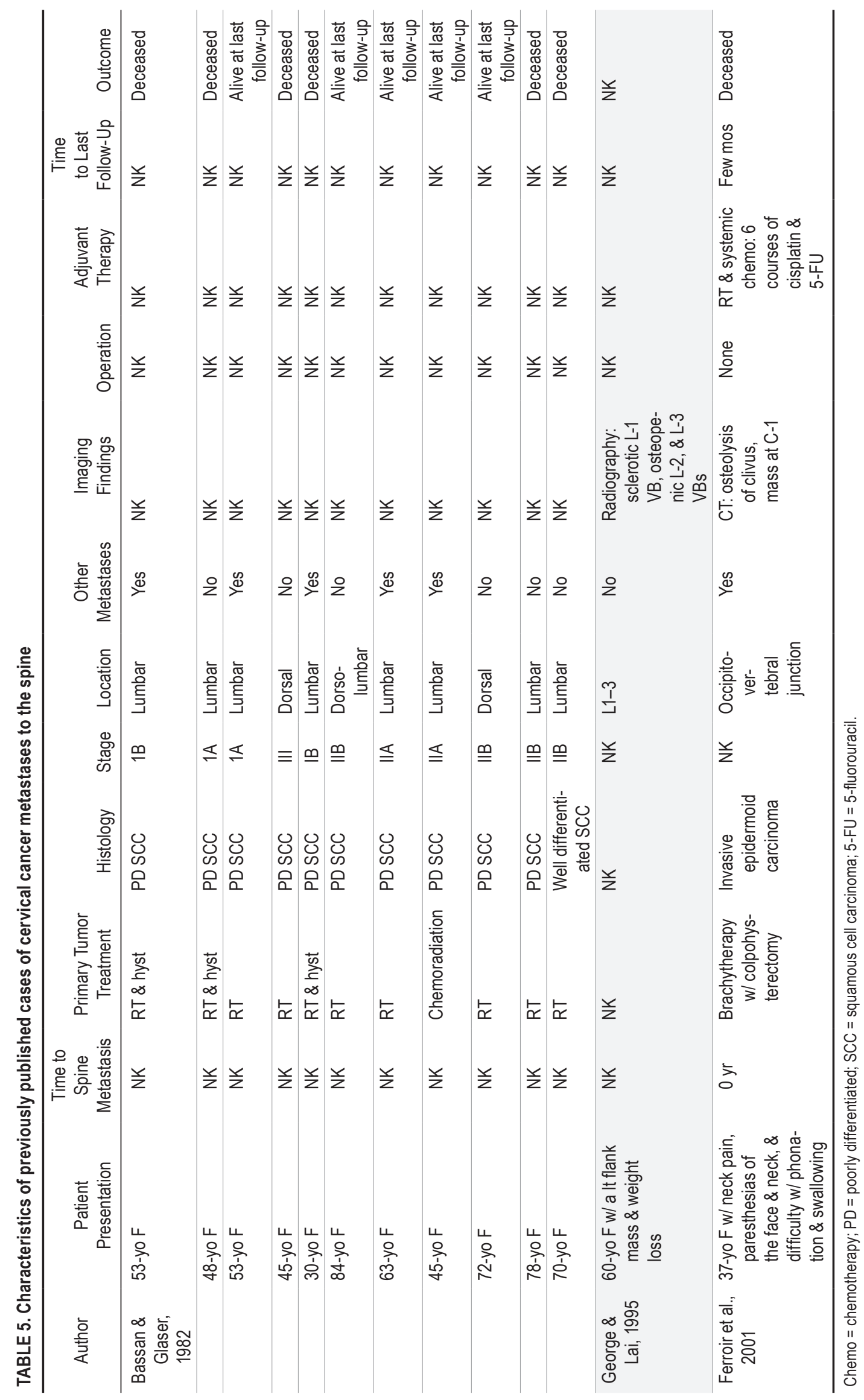




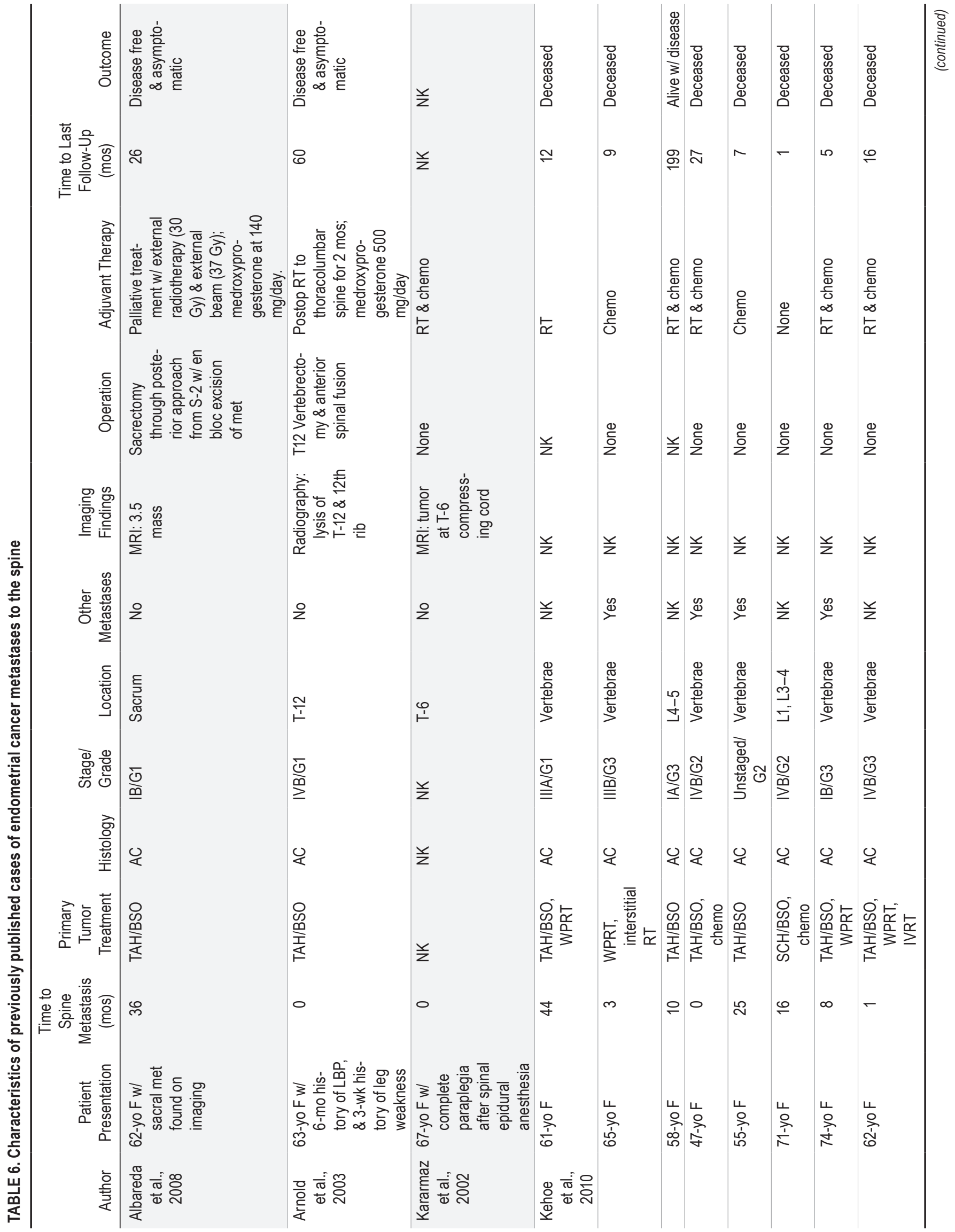




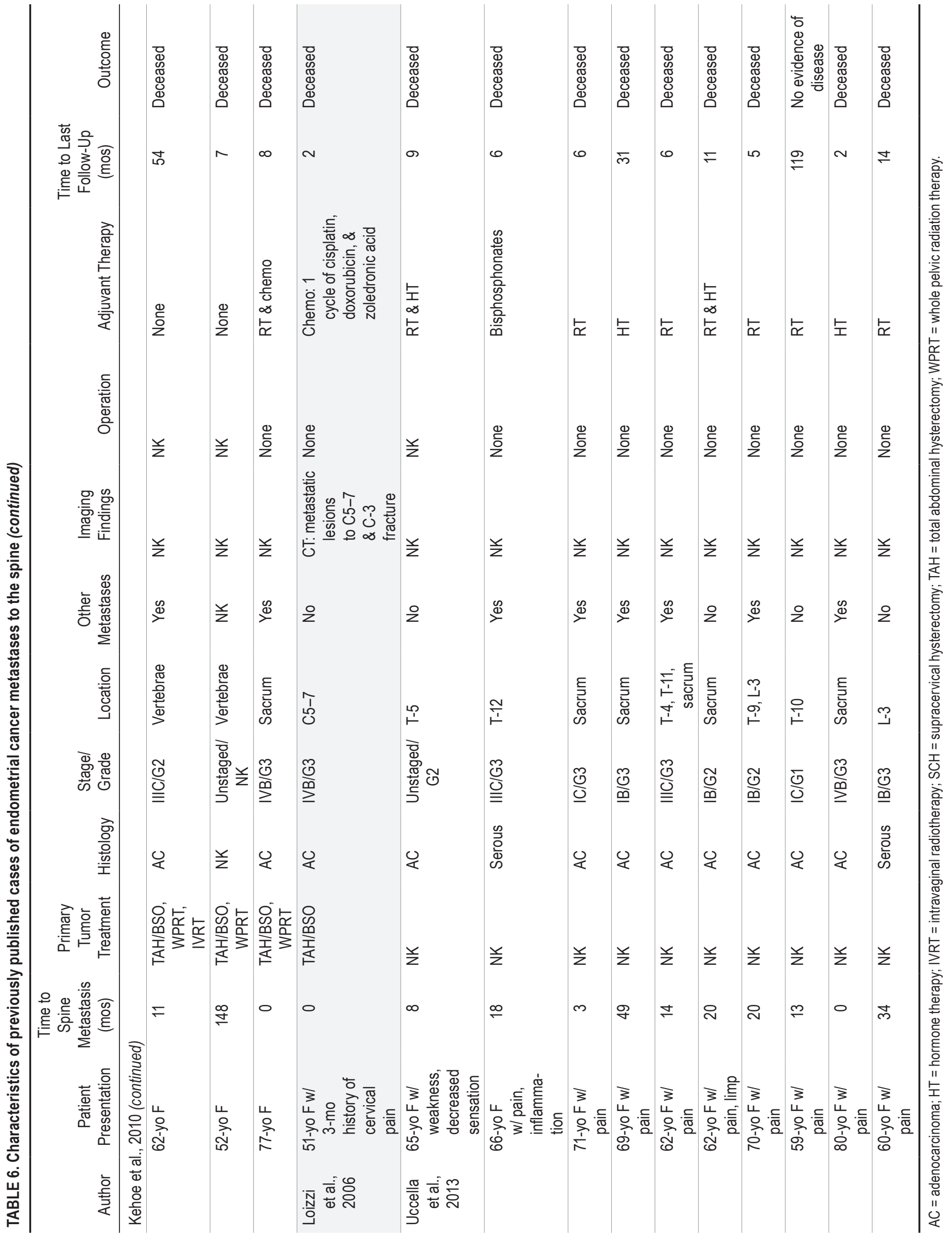




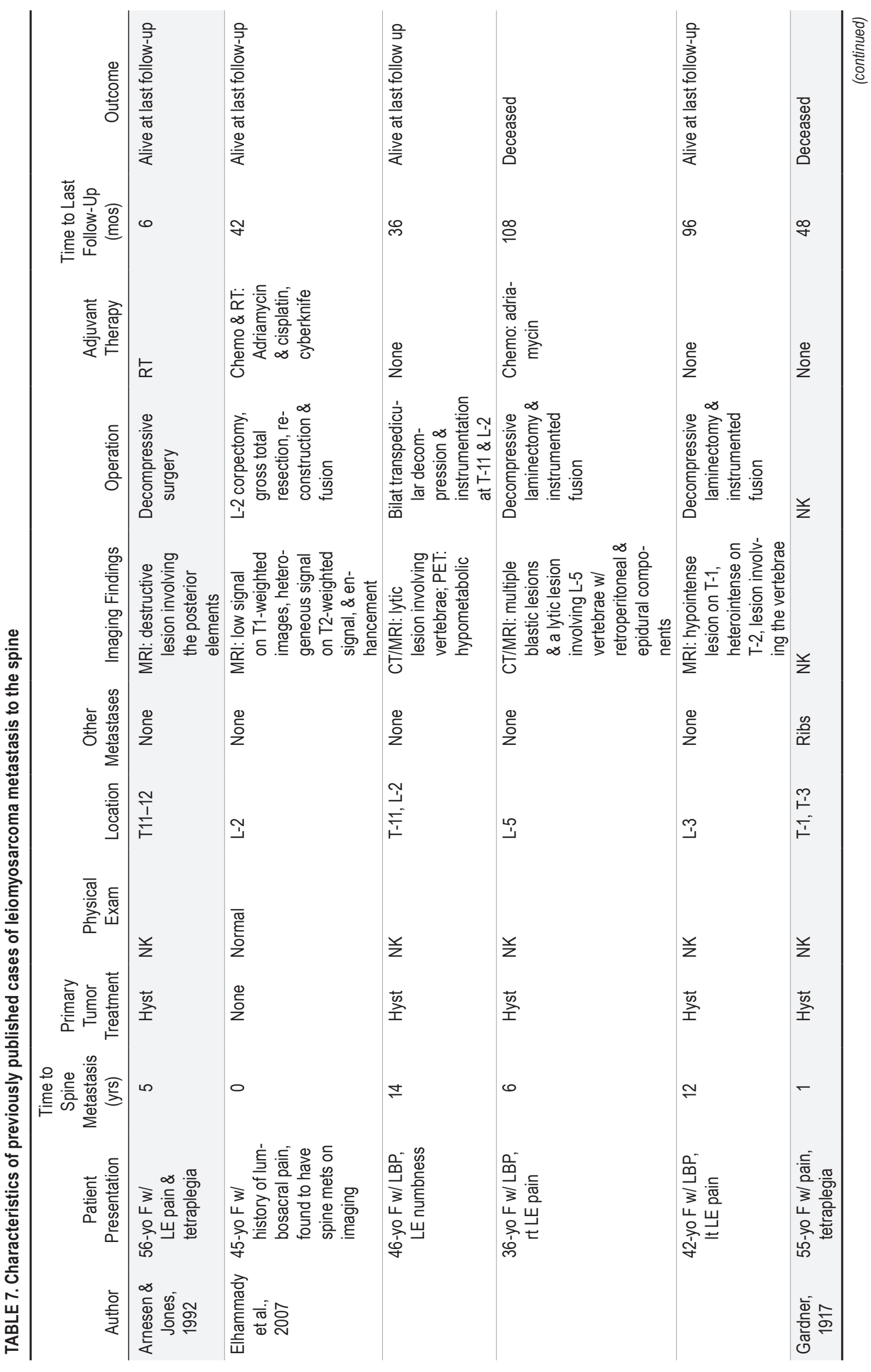




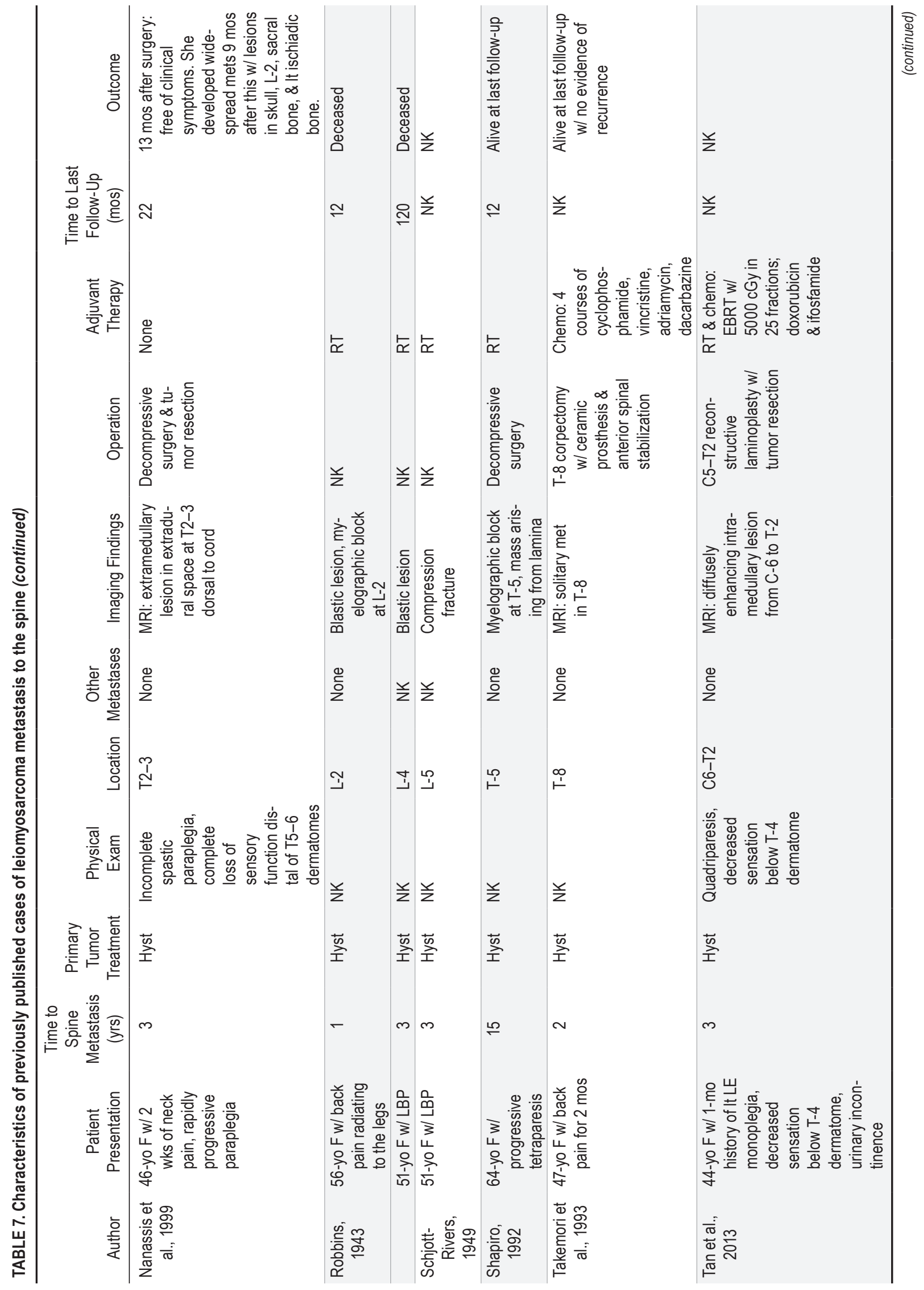




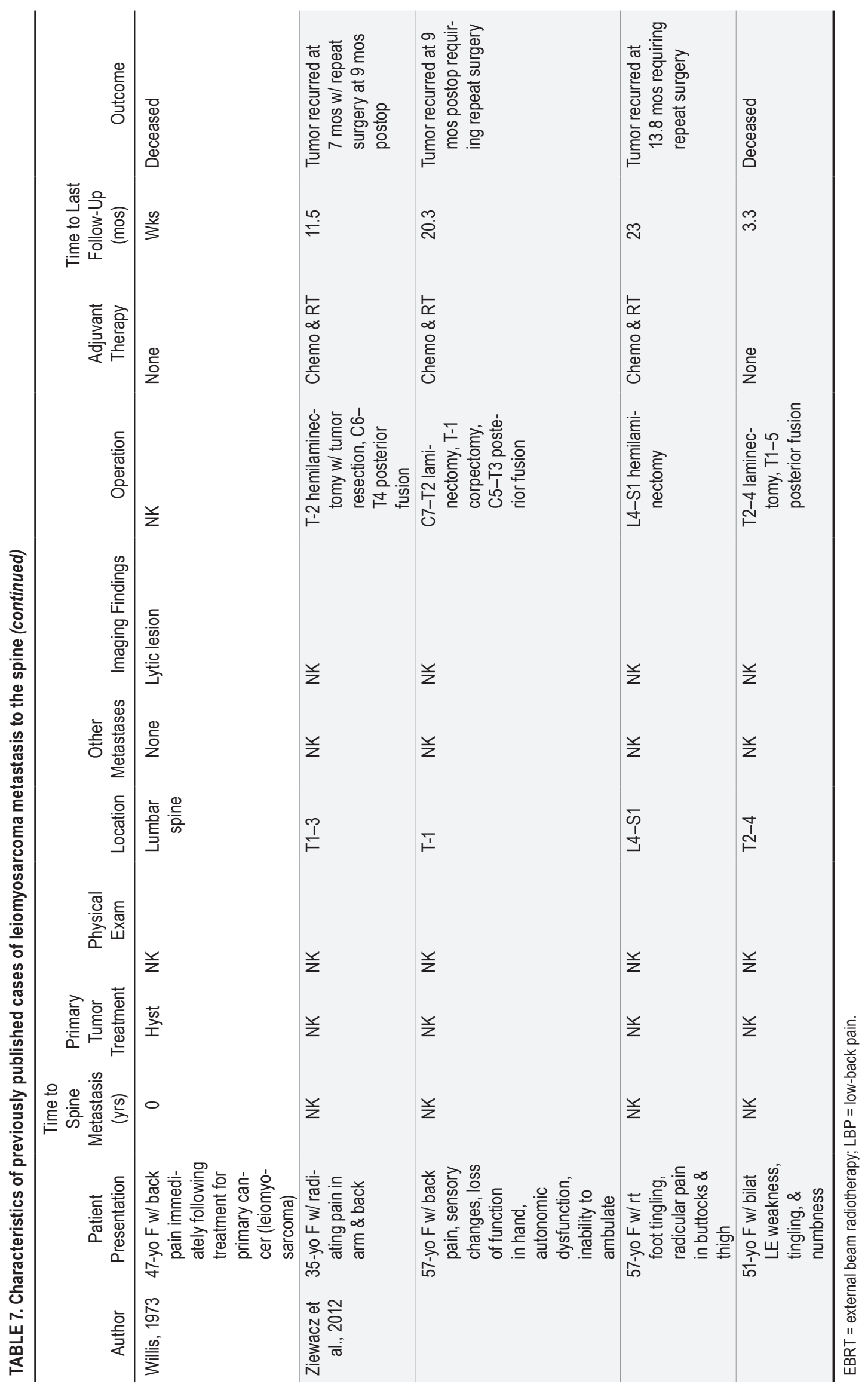




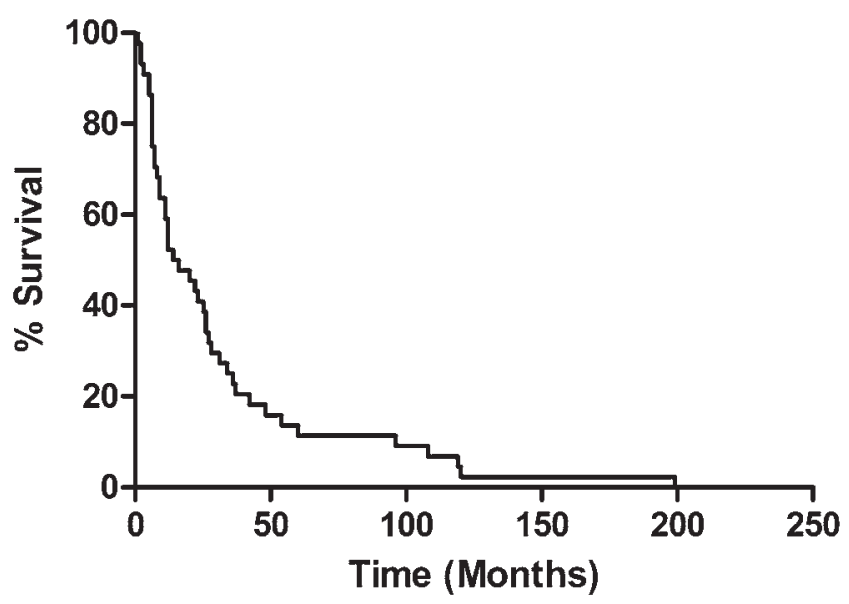

FIG. 1. Graph showing overall survival of all patients with gynecological metastases to the spine.

bony metastases ranges from 2 to 7 months..$^{10,23,28,35}$ From case reports (Table 5), 6 (46\%) of 13 reported patients were alive at last follow-up, but survival rates at specific time points could not be calculated as length of survival was not reported in these cases.

\section{Surgery for Spinal Metastasis From Endometrial Cancer}

The majority of endometrial spine metastases are presented as case reports or case series (Table 6). ${ }^{1,3,17,18,21,36}$ Based on these studies, there appears to be no predilection of location within the spine, and treatment is typically nonsurgical. Prognosis is similarly poor, with the majority of patients dying from their disease, with a median survival in the literature of 6-9 months after diagnosis of spine metastasis. ${ }^{18,36}$ From the reported literature cases alone (Fig. 2), 1-year and 5-year survival rates were $38 \%$ and $8.3 \%$, respectively, with an overall median survival of 9 months. Our patients with endometrial spine lesions survived for a median of 26 months after discovery of their spine metastasis. Of note, our series showed a substantially larger blood loss with such lesions compared with the cases of cervical cancer and leiomyosarcoma. Such a finding can likely be explained by the high vascularity of the primary organ itself, namely the endometrium, and thus concern for increased blood loss should be expected when operating on such lesions.

\section{Surgery for Spinal Metastasis From Leiomyosarcoma}

Leiomyosarcoma metastases to the spine have been well-described, affecting younger patients and having a predilection for the thoracic or lumbar spine.,38 Our patients had a mean age of 44 years, which is younger than the mean age of 50.9 and 53.8 years as described by Elhammady et al. and Ziewacz et al., respectively., ${ }^{9,38}$ Previously reported survival ranges from weeks to 13 years ${ }^{9}$ (Table 7) and generally seems to be longer than that for other gynecological malignancies. One-year and 5-year survival of patients from case reports (Fig. 2) is $64 \%$ and $21 \%$, respectively, with an overall median survival of 22.5 months. In our series, patients with leiomyosarcoma had

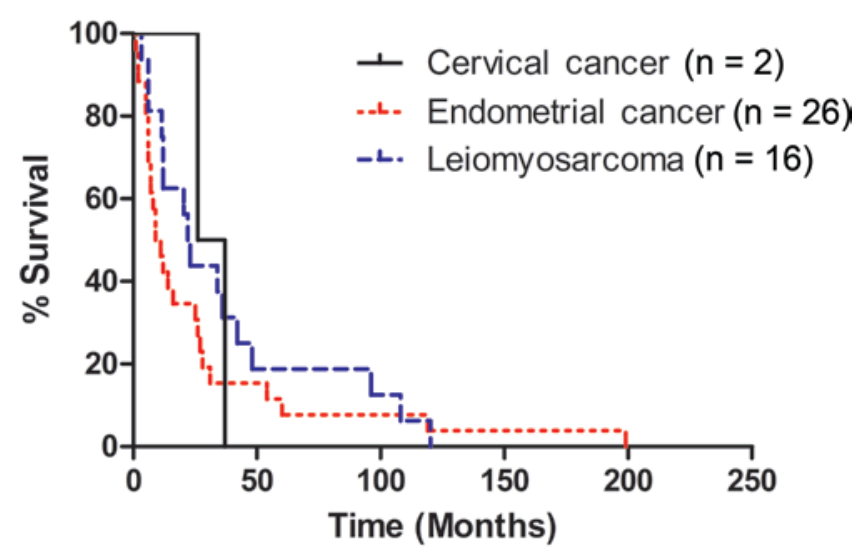

FIG. 2. Graph showing survival of patients with gynecological metastases to the spine by cancer type. For cervical cancer, survival is based on the 2 patients in our case series; the 13 cases found in the literature did not report survival and were excluded. For endometrial cancer, survival was calculated from our 2 cases as well as 24 cases from the literature with known survival; 1 case from the literature was excluded. For leiomyosarcoma, survival was calculated from our 2 cases and 14 cases from the literature; 4 cases from the literature were excluded. Figure is available in color online only.

the shortest survival, dying 20 months after spine metastasis diagnosis. This may be due to the fact that our patients had widespread metastases at the time of diagnosis. As has been previously shown, ${ }^{9,38}$ surgery with intralesional resection and stabilization improved pain and neurological function in our patients.

\section{Surgical Outcomes for All Gynecological Cancers}

In all of our patients with spine metastasis secondary to gynecological cancer, surgery was safe and without postoperative complications. All of our patients with known follow-up had stable or improved neurological outcomes, performance status, and improved pain, without local recurrence of tumor. Due to the limited number of cases in our study, the variation in survival as compared with the literature may be due to several factors such as differences in the grading, stage, and treatment of the primary tumor; involvement of the spine metastases; and baseline health of the patients at presentation. Another limitation of our study is that due to its retrospective nature, formal quality of life measures via instruments like the SF-36 or QoL5 could not be obtained and evaluated.

Spinal metastases of gynecological cancer are relatively rare, and because of this, prior reports are generally described within the context of all bony metastases, regardless of location. Additionally, few reports exist on the surgical outcomes for these patients, and thus the survival, complications, and patient satisfaction following surgery for spinal metastases from such malignancies are not clearly defined. Although our experience shows that surgery can be effective in improving pain and neurological function in a small number of patients with gynecological metastases to the spine, further prospective studies that include formal quality of life measures are needed to understand the outcomes following surgery for patients affected by these rare lesions. 


\section{Conclusions}

Gynecological cancers rarely metastasize to the spine. Combining such information with other preoperative factors may more accurately aid in surgeon management of these rare spinal lesions. When combined with previously reported cases in the literature, overall survival of all patients following diagnosis of gynecological metastasis to the spine was 15 months. Survival differs depending on primary histology, with decreasing survival from cervical cancer (32 months) to leiomyosarcoma (22.5 months) to endometrial cancer (10 months).

\section{References}

1. Albareda J, Herrera M, Lopez Salva A, Garcia Donas J, Gonzalez R: Sacral metastasis in a patient with endometrial cancer: case report and review of the literature. Gynecol Oncol 111:583-588, 2008

2. Arnesen MA, Jones JW: Spindle cell neoplasm of the thoracic spine. Ultrastruct Pathol 16:29-34, 1992

3. Arnold J, Charters D, Perrin L: Prolonged survival time following initial presentation with bony metastasis in stage IVb endometrial carcinoma. Aust N Z J Obstet Gynaecol 43:239-240, 2003

4. Barmeir E, Langer O, Levy JI, Nissenbaum M, DeMoor NG, Blumenthal NJ: Unusual skeletal metastases in carcinoma of the cervix. Gynecol Oncol 20:307-316, 1985

5. Bassan JS, Glaser MG: Bony metastasis in carcinoma of the uterine cervix. Clin Radiol 6:623-625, 1982

6. Berger AC: Introduction: role of surgery in the diagnosis and management of metastatic cancer. Semin Oncol 35:98-99, 2008

7. Centers for Disease Control: Get the Facts About Gynecologic Cancer. (http://www.cdc.gov/cancer/ knowledge/pdf/CDC_GYN_Comprehensive_Brochure.pdf) [Accessed June 29, 2015]

8. Ducimetière F, Lurkin A, Ranchère-Vince $D$, Decouvelaere AV, Péoc'h M, Istier L, et al: Incidence of sarcoma histotypes and molecular subtypes in a prospective epidemiological study with central pathology review and molecular testing. PLoS One 6:e20294, 2011

9. Elhammady MS, Manzano GR, Lebwohl N, Levi AD: Leiomyosarcoma metastases to the spine. Case series and review of the literature. J Neurosurg Spine 6:178-183, 2007

10. Fagundes H, Perez CA, Grigsby PW, Lockett MA: Distant metastases after irradiation alone in carcinoma of the uterine cervix. Int J Radiat Oncol Biol Phys 24:197-204, 1992

11. Ferroir JP, Le Breton C, Khalil A, Antoine JM, Ponnelle $\mathrm{T}$, Billy C, et al: Cranial nerve palsy revealing an occipitovertebral metastasis from carcinoma of the uterine cervix. Joint Bone Spine 68:170-174, 2001

12. Fisher MS: Lumbar spine metastasis in cervical carcinoma: a characteristic pattern. Radiology 134:631-634, 1980

13. Gabriel K, Schiff D: Metastatic spinal cord compression by solid tumors. Semin Neurol 24:375-383, 2004

14. Gardner LU: A case of metastatic leiomyosarcoma primary in the uterus. J Med Res 36:19-30, 30.1-30.3, 1917

15. George J, Lai FM: Metastatic cervical carcinoma presenting as psoas abscess and osteoblastic and lytic bony metastases. Singapore Med J 36:224-227, 1995

16. Hage WD, Aboulafia AJ, Aboulafia DM: Incidence, location, and diagnostic evaluation of metastatic bone disease. Orthop Clin North Am 31:515-528, vii, 2000

17. Kararmaz A, Turhanoglu A, Arslan H, Kaya S, Turhanoglu S: Paraplegia associated with combined spinal-epidural anaesthesia caused by preoperatively unrecognized spinal vertebral metastasis. Acta Anaesthesiol Scand 46:11651167,2002

18. Kehoe SM, Zivanovic O, Ferguson SE, Barakat RR, Soslow RA: Clinicopathologic features of bone metastases and outcomes in patients with primary endometrial cancer. Gynecol Oncol 117:229-233, 2010

19. Kim RY, Weppelmann B, Salter MM, Brascho DJ: Skeletal metastases from cancer of the uterine cervix: frequency, patterns, and radiotherapeutic significance. Int J Radiat Oncol Biol Phys 13:705-708, 1987

20. Lengyel E: Ovarian cancer development and metastasis. Am J Pathol 177:1053-1064, 2010

21. Loizzi V, Cormio G, Cuccovillo A, Fattizzi N, Selvaggi L: Two cases of endometrial cancer diagnosis associated with bone metastasis. Gynecol Obstet Invest 61:49-52, 2006

22. Mariani A, Webb MJ, Keeney GL, Calori G, Podratz KC: Hematogenous dissemination in corpus cancer. Gynecol Oncol 80:233-238, 2001

23. Matsuyama T, Tsukamoto N, Imachi M, Nakano H: Bone metastasis from cervix cancer. Gynecol Oncol 32:72-75, 1989

24. Nanassis K, Alexiadou-Rudolf C, Tsitsopoulos P: Spinal manifestation of metastasizing leiomyosarcoma. Spine (Phila Pa 1976) 24:987-989, 1999

25. National Cancer Institute: SEER Stat Fact Sheets: Cervix Uteri Cancer. (http://seer.cancer.gov/statfacts/html/cervix. html) [Accessed June 29, 2015]

26. National Cancer Institute: SEER Stat Fact Sheets: Endometrial Cancer. (http://seer.cancer.gov/statfacts/html/ corp.html) [Accessed June 29, 2015]

27. National Cancer Institute: SEER Stat Fact Sheets: Ovary Cancer. (http://seer.cancer.gov/statfacts/html/ovary.html) [Accessed June 29, 2015]

28. Ratanatharathorn V, Powers WE, Steverson N, Han I, Ahmad $\mathrm{K}$, Grimm J: Bone metastasis from cervical cancer. Cancer 73:2372-2379, 1994

29. Robbins LL: Roentgenologic demonstration of spinal metastases from leiomyosarcoma of the uterus. Arch Surg 47:463-467, 1943

30. Schjott-Rivers E: Sarcoma of the uterus. Acta Obstet Gynecol Scand 28:418-425, 1949

31. Shapiro S: Myelopathy secondary to leiomyosarcoma of the spine. Case report. Spine (Phila Pa 1976) 17:249-251, 1992

32. Siegel R, Naishadham D, Jemal A: Cancer statistics, 2012. CA Cancer J Clin 62:10-29, 2012

33. Takemori M, Nishimura R, Sugimura K, Mitta M: Thoracic vertebral bone metastasis from uterine leiomyosarcoma. Gynecol Oncol 51:244-247, 1993

34. Tan LA, Kasliwal MK, Nag S, O’Toole JE: A rare intramedullary spinal cord metastasis from uterine leiomyosarcoma. J Clin Neurosci 20:1309-1312, 2013

35. Thanapprapasr D, Nartthanarung A, Likittanasombut $\mathrm{P}, \mathrm{Na}$ Ayudhya NI, Charakorn C, Udomsubpayakul U, et al: Bone metastasis in cervical cancer patients over a 10 -year period. Int J Gynecol Cancer 20:373-378, 2010

36. Uccella S, Morris JM, Bakkum-Gamez JN, Keeney GL, Podratz KC, Mariani A: Bone metastases in endometrial cancer: report on 19 patients and review of the medical literature. Gynecol Oncol 130:474-482, 2013

37. Willis RA: The Spread of Tumours in the Human Body, ed 3. London: Butterworths, 1973, p 234

38. Ziewacz JE, Lau D, La Marca F, Park P: Outcomes after surgery for spinal metastatic leiomyosarcoma. J Neurosurg Spine 17:432-437, 2012

\section{Disclosure}

Ms. Liu reports being a Howard Hughes Medical Institute 
Research Fellow. Dr. Goodwin reports being a UNCF Merck postdoctoral fellow and receiving an award from the Buroughs Wellcome Fund. Dr. Witham reports receiving support from Eli Lilly and Company and the Gordon and Marilyn Macklin Foundation for non-study-related clinical or research effort as well as honoraria from AO Spine North America for CME courses. Dr. Bydon reports receiving a research grant from DePuy Spine and serving on the clinical advisory board of MedImmune, LLC. Dr. Gokaslan reports stock ownership in US Spine and Spinal Kinetics; consulting, speaking, and teaching for the AO Foundation; and receiving research support from DePuy, NREF, AOSpine, and AO North America. Dr. Sciubba reports being a consultant for DePuy Synthes, Medtronic, NuVasive, Stryker, and Globus.

\section{Author Contributions}

Conception and design: Sciubba, Liu, Sankey, Goodwin. Acquisi- tion of data: Sciubba, Liu, Sankey, Bydon, Witham, Wolinsky, Gokaslan. Analysis and interpretation of data: Sciubba, Liu, Sankey, Goodwin. Drafting the article: Sciubba, Liu, Sankey, Goodwin. Critically revising the article: Sciubba, Liu, Sankey, Goodwin, Kosztowski, Elder. Reviewed submitted version of manuscript: all authors. Approved the final version of the manuscript on behalf of all authors: Sciubba. Statistical analysis: Liu, Sankey. Administrative/technical/material support: Sciubba.

Study supervision: Sciubba, Goodwin, Elder, Bydon, Witham, Wolinsky, Gokaslan.

\section{Correspondence}

Daniel M. Sciubba, Department of Neurosurgery, Johns Hopkins University School of Medicine, 600 North Wolfe Street, Meyer 7-109, Baltimore, MD 21287. email: dsciubb1@jhmi.edu. 